\title{
Inflammatory and fibrosis infiltration in synovium associated with the progression in developmental dysplasia of the hip
}

\author{
DE LI ${ }^{*}$, HUI WANG ${ }^{*}$, JI-YE HE, CHENG-LONG WANG, WEI-JIA FENG, CHAO SHEN, \\ JUN-FENG ZHU, DONG-LIANG WANG and XIAO-DONG CHEN \\ Department of Orthopedic Surgery, Xin-Hua Hospital, \\ Shanghai Jiao-Tong University School of Medicine, Shanghai 200092, P.R. China
}

Received February 12, 2018; Accepted June 13, 2018

DOI: $10.3892 / \mathrm{mmr} .2019 .9910$

\begin{abstract}
Developmental dysplasia of the hip (DDH) is a common musculoskeletal disorder characterized by progressive joint soreness and limited mobility. The aim of the present study was to investigate the pathological changes and inflammatory infiltration in the hypertrophic synovium of the hip joint associated with the progression of DDH. Synovial biopsies in the hip joint are obtained from patients with moderate DDH and severe DDH during surgery. These biopsies are processed for histological and immunohistochemical (IHC) analysis and investigation of the pathological processes in a synovium, including types of inflammatory cell infiltration, synovial angiogenesis and fibrosis, neuron endings and neuropeptide invasion. Correlation analysis was performed between the mean optical density (MOD) of each antibody, and Harris hip score (HHS) and visual analogue score (VAS)
\end{abstract}

Correspondence to: Dr Dong-Liang Wang or Dr Xiao-Dong Chen, Department of Orthopedic Surgery, Xin-Hua Hospital, Shanghai Jiao-Tong University School of Medicine, 1665 Kongjiang Road, Shanghai 200092, P.R. China

E-mail: wangdongliang@xinhuamed.com.cn

E-mail: chenxiaodong@xinhuamed.com.cn

*Contributed equally

Abbreviations: DDH, developmental dysplasia of the hip; CGRP, calcitonin gene-related peptide; SP, substance P; IHC, immunohistochemistry; Elisa; MMPs, matrix metalloproteinases; IL, interleukin; PAO, periacetabular osteotomy; THA, total hip arthroplasty; OA, osteoarthritis; MOD, mean optical density; HHS, harris hip score; VAS, visual analogue score; NF-200, Neurofilament-200; CDH, congenital dysplasia of the hip; ESR, erythrocyte sedimentation rate; CRP, C-reactive protein; CEA, centre edge angle; LCA, leukocyte common antigen; MIB1, mindbomb homolog 1; $\alpha$-SMA, $\alpha$ smooth muscle actin; DRG, dorsal root ganglion; ECM, extracellular matrix; MCP-1, monocyte chemotactic protein-1; MIP-1 $\beta$, macrophage inflammatory protein-1; MCT, mast cell tryptase

Key words: synovium, fibrosis, osteoarthritis, dysplasia using the Spearman correlation test. Chronic inflammation in the synovium was observed via the positive IHC staining of inflammatory cells, such as T cells, B cells, macrophages and leukocytes. Excessive staining of vimentin and $\alpha$ smooth muscle actin in the synovium of severe DDH represented significant fibrosis and angiogenesis. These targets were also significantly correlated with HHS in severe DDH. The MOD levels of CD68 (indicators of macrophage) indicated apparent correlations with HHS and VAS in patients with severe DDH. The labels of nerve fibers and pain transmission indicators were as follows: Neurofilament-200 and substance P. Calcitonin gene-related peptide was upregulated in the synovium of severe DDH in contrast to that in the synovium of moderate DDH. The MOD levels of NF-200, SP and CGRP were correlated with VAS in severe DDH. The pathology of DDH includes chronic inflammatory cell infiltration corresponding with nerve fibers and fibroblastic proliferation, which might contribute to arthritis progression and joint soreness in DDH.

\section{Introduction}

Developmental dysplasia of the hip (DDH), formerly known as congenital dysplasia of the hip (CDH) affects $1-5 \%$ babies in China and has a female-to-male ratio of 4-10:1 (1,2). Shallow acetabulum, slacking joint capsule, and narrowing joint space are the main anatomical features of $\mathrm{DDH}$, which result in chronic pain, joint stiffness, synovial inflammation, and hyperplasia in fossa acetabuli.

As the hallmark symptom of DDH, the pain experience is well-recognized as typically transitioning from an intermittent weight-bearing pain to an increasingly persistent and chronic pain around the hip joint. Besides, the characteristic pain in $\mathrm{DDH}$ is increasing restriction of active and passive movement. The hip joint becomes stiff with the progression of DDH, and this stiffness is usually observed in the later stage of DDH and worsens with the progression of osteoarthritis (OA).

Previous theories demonstrated that the initial hip pain in OA is mainly derived from acetabular labral lesions (3-5), followed by the infiltration of sensory neurons into the synovium and labrum $(6,7)$. However, OA is a multifactorial inducing disease, which is not commonly believed as mainly secondary from the progress of DDH. Additionally, the development of OA is usually a long history, but the phenomenon 
of many young adult patients with moderate DDH (slight pain and joint restriction) (Fig. 1A) developing into severe DDH (acute pain and joint dysfunction) (Fig. 1B) within a few years was frequently observed in clinic, which is not the characteristic process of OA. Therefore, this phenomenon prompts us to investigate the underlying pathologic changes during the development of DDH. As we know, moderate or severe DDH is characterized by cartilage degeneration, synovial hyperplasia, subchondral sclerosis, inflammation and osteophyte formation. So we speculated that immune cells and inflammatory cells are derived from two aspects: Peripheral blood and subchondral bone. On the one hand, the abnormal stress may promote subchondral bone to release chemotaxis signals. T cells, B cells, macrophages induced by the signals migrate to hip joint synovium. Inflammatory mediators are secreted by the cells into the hip joint cavity, causing the destruction of articular cartilage. On the other hand, the abnormal stress of subchondral bone force the immune cells and stem cells which come from the bone marrow to move to osteochondral junction. Chemokines and MMPs are released and cause osteochondral destruction. The aim of this study is mainly to observe the pathological changes and inflammatory infiltration in the hypertrophic synovium of hip joint associated with the progression of $\mathrm{DDH}$ and to investigate the effect of synovial hyperplasia on cartilage degeneration.

Synovial membrane inflammation in OA is usually secondary to adjacent cartilage degradation and characterized by angiogenesis, invasion of immune cells and pro-inflammatory mediators. Mast cells are widely distributed around the microvasculature of the articular synovium, skin and visceral mucosa which can secrete a variety of cytokines and are involved in immune regulation such as T cells, B cells and APC cells activation. Synovial inflammation is correlated with the severity and progress of OA and is also the main cause of pain in patients with OA $(8,9)$. Recently, research on pain and stiffness in musculoskeletal disorders has been advanced with histological and immunohistochemical (IHC) analysis of synovium, which has been increasingly useful in identifying pathological changes in vivo $(10,11)$. Analysis of synovial tissues in OA represents inflammatory changes and neuron infiltration $(12,13)$, whereas synovium from frozen shoulders display not only inflammatory and angiogenesis but also fibrosis and proliferative myelofibrosis $(14,15)$. Undoubtedly, considerable cytokines might become involved in the development of DDH, but the pathology of this process remains unclear, especially in different stages of DDH. Thus, the purpose of this study is to identify the cell type involved in synovium of DDH by using novel IHC analysis and antibodies and improve our understanding of pathophysiologic progression in DDH.

\section{Materials and methods}

Patients and biopsies. This study was carried out in accordance with the Declaration of Helsinki and was approved by the ethics committee of Xinhua Hospital affiliated to Shanghai Jiao Tong University School of Medicine. The samples obtained from the operations were prepared for testing purposes only and with the approval and signed consent of the patients.
The clinical biopsies used in this study were obtained from patients who accepted surgical treatments in our institution between November 2013 and December 2014. The patients with DDH involved in this study were divided into two groups according to their clinical diagnoses and severities (16). A total of 45 patients $(\mathrm{F} / \mathrm{M}=38 / 7$, mean age $=26.5$ years $)$ with DDH (Crowe: I-II) and grade 2 or less OA of K-L classification (Kellgren and Lawrence Scale) were set as the moderate DDH group. A total of 35 patients $(\mathrm{F} / \mathrm{M}=27 / 8$, mean age $=29.6$ years $)$ with DDH (Crowe: I-II) and grade 3-4 OA of K-L classification were set as the severe DDH group. The degrees of hip joint dislocation and the severities of OA were divided according to the classification of Crowe and the grade of K-L, respectively, in clinic $(17,18)$. Clinical and laboratory data for the entire groups are presented in Table I. Variables, such as erythrocyte sedimentation rate (ESR) and C-reactive protein (CRP), were measured by a standard clinical technology. The patients in two groups were matched according to gender and age and absence of systemic disease.

The diagnosis of DDH was based on the standard anteroposterior radiograph of hip with a sharp angle of $<45^{\circ}$ and a center edge angle (CEA) of $<20^{\circ}(19,20)$. The patients with moderate DDH received operation of periactabular osteotomy (PAO), whereas those with severe DDH underwent total hip arthroplasty (THA). The hyperplasia synovium was extracted from the inner wall of the joint capsule when we opened the joint capsule during the operation.

IHC analysis. Synovial specimens obtained from these patients during the operation were immediately cut into pieces of $1 \mathrm{~cm}^{3}$ and immersed in $4 \%$ paraformaldehyde for $24 \mathrm{~h}$, then washed, dehydrated with a graded series of alcohol solutions, and finally embedded in paraffin for $24 \mathrm{~h}$. The blocks were cut into $5 \mu \mathrm{m}$ sections with a Leica microtome (RM2255; Leica Microsystems GmbH, Wetzlar, Germany) and were mounted on anti-off slides (no. 4951PLUS; Thermo Fisher Scientific, Inc., Waltham, MA, USA).

The slides were stained with hematoxylin and eosin for histological analysis. After citrate antigen retrieval and peroxidase inactivation treatment, immunohistochemistry was performed with antibodies directed against CD45 (Leukocyte common antigen; LCA), CD3 (T cells), CD20 (B cells), CD68 (macrophages), neurofilament-200 (NF-200; neural marker), mindbomb homolog 1 (MIB1) (proliferative cell markers), substance P (SP; neuropeptides), calcitonin gene-related peptide (CGRP; neuropeptides), vimentin (fibroblasts), $\alpha$ smooth muscle actin ( $\alpha$-SMA; myofibroblasts), and mast cell tryptase (mast cells). The slides were rinsed in phosphate buffer saline (3x5 min) and incubated with biotinylated secondary antibody (Dako, Ely, UK). Finally, 3'-diaminobenzidine tetra-chloride) was used for the visualization of immunoreactions.

The images of the microscopic fields were captured by a camera (DEI750; Optronics Engineering, Goleta, CA, USA) attached to the microscope. In each biopsy, two slides were obtained at different depths, and eight microscopic fields from each slide were photographed and analyzed. Thus, in every synovial sample, 16 microscopic fields were collected. The mean optical density (MOD) levels were measured with Image-Pro plus 6.0 (Media Cybernetics, Inc., Rockville, MD, USA) as previously described (21). The results were expressed 

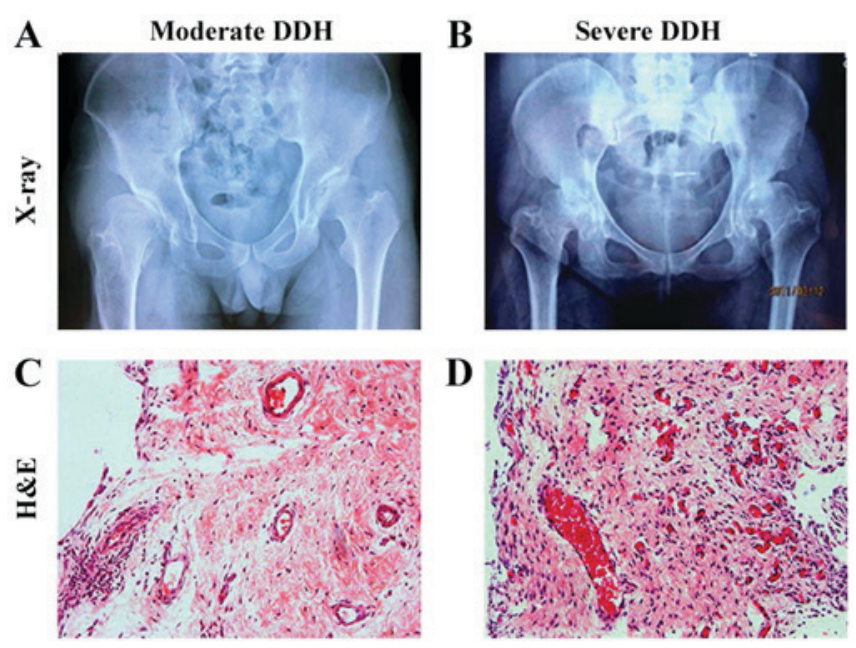

Figure 1. Radiographs of hip joint and H\&E staining of synovium from different DDH groups. Pelvic radiograph from (A) moderate DDH and (B) severe DDH. (A) shows apparent joint space and mild osteoarthritis; (B) shows narrow joint space and severe osteoarthritis. (C and D) H\&E staining of synovium slides. Magnification, x200. DDH, developmental dysplasia of the hip; H\&E, hematoxylin and eosin.

as the positive staining density in relation to the total area of each microscopic field.

All these slides were detected under light microscopy, and the presence of labeled cells is documented and tabulated in Table II. The absence of staining was documented as a negative result (-), and the presence of staining was noted as a positive result $(+)$ by using a scale based on the number of cells per high-power field $(\mathrm{x} 400),(+), 1$ to $4 ;(++), 5$ to $10 ;(+++)$, $>10$; and (++++), >100 (14).

Statistics. The results in Table I are expressed as mean \pm standard deviation (SD). The comparisons the between moderate and severe DDH groups with respect to the MOD levels of each antibody in the synovium were preformed using the Mann-Whitney U test. Graphpad software (version 5.01; GraphPad Software, Inc., La Jolla, CA, USA) was used. A $\mathrm{P}<0.05$ was considered to indicate a statistically significant difference. The correlations analyses were performed with Spearman rank correlation test. A two-sided P-value $<0.01$ was statistically significant.

\section{Results}

Imaging, histological and IHC analysis. Pelvic radiograph illustrate the morphology of the femoral head and the acetabulum of the patients with moderate DDH to severe DDH (Fig. 1A and B). Histological analysis of the synovial tissue revealed evidence of varied degrees of chronic inflammation, the hyperplasia of synovial layers, and infiltration of inflammatory cells (Fig. 1 and Table II) during the process from moderate DDH to severe DDH.

The immunohistochemistry stains showed macrophage infiltration and proliferation of inflammatory cells through the special positive staining of CD68 (macrophage), CD3 (T cells), CD45 (LCA), CD20 (B cells), and mast cell tryptase (mast cells). The result of IHC staining implied that the CD68 expression in the synovial tissue of severe DDH was higher
Table I. Data and clinical details of patients and normal people recruited in this study.

\begin{tabular}{lcc}
\hline Characteristics & Moderate DDH & Severe DDH \\
\hline Number & 45 & 35 \\
Ages & $26.5 \pm 6.1$ & $29.6 \pm 4.9$ \\
Female/Male & $38 / 7$ & $27 / 8$ \\
BMI (kg/m ${ }^{2}$ ) & $22.8 \pm 1.9$ & $22.8 \pm 2.5$ \\
Durations (years) & $4.6 \pm 1.5$ & $3.8 \pm 2.1$ \\
CRP (mg/l) & $9.9 \pm 3.1$ & $9.8 \pm 3.4$ \\
ESR (mm/1st h) & $19.1 \pm 3.9$ & $20.7 \pm 6.1$ \\
Harris hip score & $67.7 \pm 8.2$ & $57.4 \pm 8.4^{\mathrm{a}}$ \\
Visual analogue score & $4.1 \pm 0.7$ & $4.4 \pm 0.9$ \\
\hline
\end{tabular}

BMI, body mass index, CRP, C-reactive protein, ESR, erythrocyte sedimentation rate. Data are given as mean \pm standard deviation. ${ }^{\mathrm{a}} \mathrm{P}<0.05$, compared to data in moderate DDH.

than that of moderate DDH (Fig. 2A-C). CD3 expression was no significant difference between moderate DDH and severe DDH (Fig. 2D-F). The positive targets were indicated with fulvous staining in each figure. The observation of nerve fibers was also confirmed by staining NF-200 (Fig. 3A and B), and the MOD of NF-200 in severe DDH was higher than that in moderate DDH (Fig. 3C). Excessive staining with vimentin, a biomarker of fibroblast, indicated that the fibroblast had an overwhelming proportion in the synovial tissues affected by severe DDH (Fig. 3D and E). The trend of vimentin coincided with NF-200 (Fig. 3F). CD20, CD45 are respectively biomarker of $\mathrm{B}$ cells and leukocytes. The increased expression of CD20, CD45 protein in the synovial tissue of patients experiencing moderate DDH or severe DDH was detected by IHC staining (Fig. 4A-F). The results indicated chronic inflammatory cells infiltration. IHC staining showed that the expression of mast cell tryptase in the synovial tissue of severe DDH was lower than that of moderate DDH (Fig. 5A-C). IHC staining showed that the expression of mast cell tryptase in the synovial tissue of severe DDH was lower than that of moderate DDH (Fig. 5A-C). Moreover, strong positive staining of MIB1 also illustrated that the active proliferation status of fibroblast (Fig. 5D-F). $\alpha$-SMA (smooth muscle cell and myofibroblast markers), which symbolized the proliferation of microvascular and myofibroblast (Fig. 5G-I) suggest excessive vascularization and fibrosis in the synovium of severe DDH compared with moderate DDH. Additionally, in severe DDH, staining of SP and CGRP in synovium were significantly up-regulated compared with those in moderate DDH (Fig. 6A-F), indicating the involvement of neuropeptides into the synovium during the development of DDH.

Comparisons of MOD levels of each targets between moderate $D D H$ and severe $D D H$. The results of MOD analysis, particularly those performed on CD68 (Fig. 2C), NF-200 (Fig. 3C), vimentin (Fig. 3F), CD45 (Fig. 4C), CD20 (Fig. 4F), $\alpha$-SMA (Fig. 5F), SP (Fig. 6C), and CGRP (Fig. 6F), presented increase in severe DDH and not in moderate DDH, and these results coincided with the results of the comparisons among the 
Table II. Results for immunocytochemical staining of biopsy material from the DDH.

\begin{tabular}{|c|c|c|c|c|c|}
\hline \multirow[b]{2}{*}{$\begin{array}{l}\text { Antibody moderate } \\
\text { DDH }\end{array}$} & \multirow[b]{2}{*}{$\begin{array}{c}\text { Cell type severe } \\
\text { DDH }\end{array}$} & \multicolumn{2}{|c|}{ Result $^{\mathrm{a}}$} & & \\
\hline & & $\begin{array}{l}\text { Moderate } \\
\text { DDH }\end{array}$ & $\begin{array}{l}\text { Severe } \\
\text { DDH }\end{array}$ & \multicolumn{2}{|c|}{ Number of patients positive } \\
\hline CD3 & T cells & + & ++ & $30 / 45$ & $29 / 35$ \\
\hline CD20 & B cells & + & +++ & $28 / 45$ & $31 / 35$ \\
\hline CD45 & Leukocytes & ++ & +++ & $38 / 45$ & $34 / 35$ \\
\hline CD68 & Macrophage & + & ++ & $34 / 45$ & $30 / 35$ \\
\hline NF-200 & Neural fiber & + & ++ & $37 / 45$ & $33 / 35$ \\
\hline Vimentin & Fibroblasts & +++ & ++++ & $43 / 45$ & $34 / 35$ \\
\hline MIB-1 & Proliferating cells & +++ & +++ & $44 / 45$ & $31 / 35$ \\
\hline$\alpha-\mathrm{SMA}$ & Myofibroblasts & + & +++ & $34 / 45$ & $35 / 35$ \\
\hline Mast cell tryptase & Mast cells & ++ & ++ & $38 / 45$ & $26 / 35$ \\
\hline \multicolumn{6}{|l|}{ Neuropeptides } \\
\hline $\mathrm{SP}$ & & + & ++ & $33 / 45$ & $33 / 35$ \\
\hline CGRP & & + & ++ & $38 / 45$ & $32 / 35$ \\
\hline
\end{tabular}

${ }^{a}$ Result, number of cells counted per high-power field $x 400 ;-$, none;,+ 1 to $4 ;++, 5$ to $10 ;+++,>10 ;++++,>100$. $\alpha$-SMA, $\alpha$-smooth muscle actin; MIB1, mindbomb homolog 1; SP, substance P; CGRP, calcitonin gene-related peptide.

A

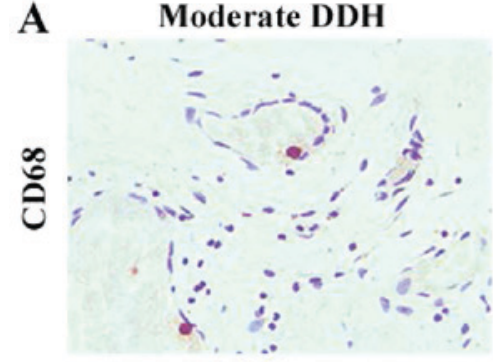

D

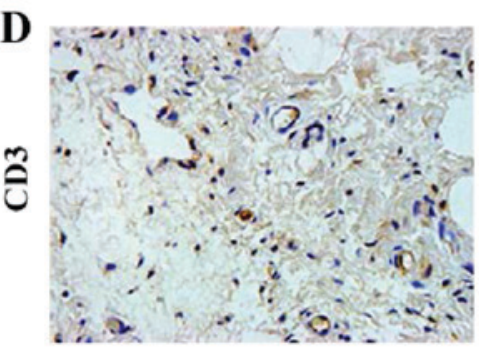

B

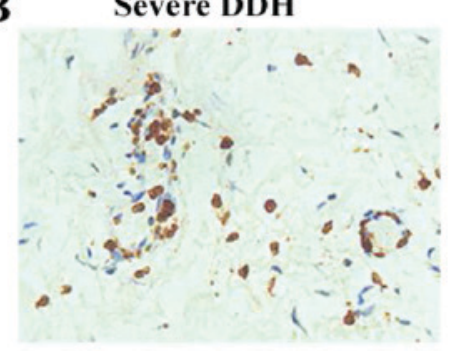

$\mathbf{E}$

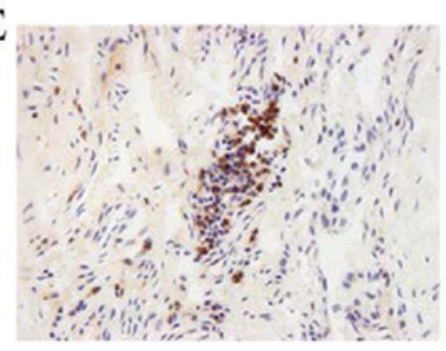

$\mathrm{C}$

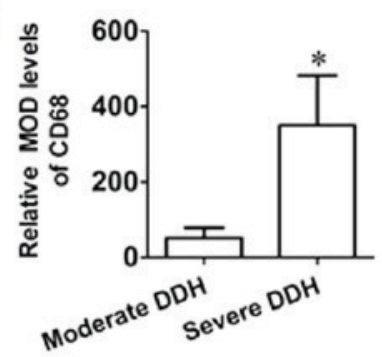

F

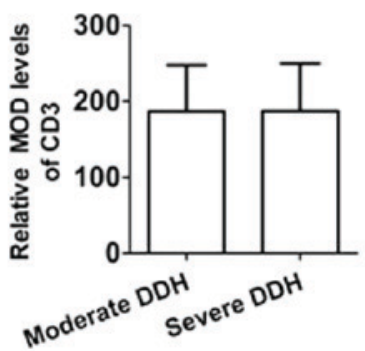

Figure 2. (A-F) Immunohistochemical staining and MOD analysis. (A, B, D and E) Immunohistochemical staining of CD68 and CD3 in synovium between moderate and severe DDH group. (A and B) Magnification: x400, (D and E) magnification: x200. (C and F) Comparisons of MOD levels of CD68 and CD3 in immunohistochemical staining between different groups. ${ }^{*} \mathrm{P}<0.05$ compared with moderate DDH. MOD, mean optical density; DDH, developmental dysplasia of the hip.

targets in IHC. However, mast cell tryptase (Fig. 5C), indicators of mast cell, presented significant decreased MOD levels in synovial of severe DDH compared with that in moderate DDH. And, the MOD levels of CD3 and MIB1 in these groups revealed approximative results with no significant difference (Fig. 2F and Fig. 5I). In addition, the presence of labeled cells is documented and tabulated in Table II.

Correlation tests of MOD levels of each antibody with Harris hip score (HHS) and visual analogue score (VAS) in patients between different groups. In correlation detection (Table III), significant correlations were observed in moderate DDH: CD45/VAS: $r=0.555, \mathrm{P}<0.01$; Vimentin/HHS: $r=-0.368, \mathrm{P}<0.01$; MCT/VAS: $r=0.571, \mathrm{P}<0.01$. The following correlations were observed in Severe DDH group: CD68/HHS: $r=-0.669, \mathrm{P}<0.01$; NF-200/VAS: $r=0.421, \mathrm{P}<0.01$; CD68/VAS: $\mathrm{r}=0.518, \mathrm{P}<0.01$; $\alpha$-SMA/HHS: $r=-0.541, \mathrm{P}<0.01$; Vimentin/HHS: $r=0.479$, $\mathrm{P}<0.01$. Additionally, neuropeptides: SP/VAS: $\mathrm{r}=0.433, \mathrm{P}<0.01$; CGRP/VAS: $\mathrm{r}=0.455, \mathrm{P}<0.01$ were also observed in the severe DDH group. 
A

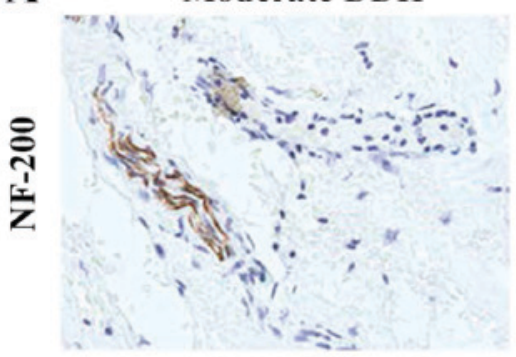

D

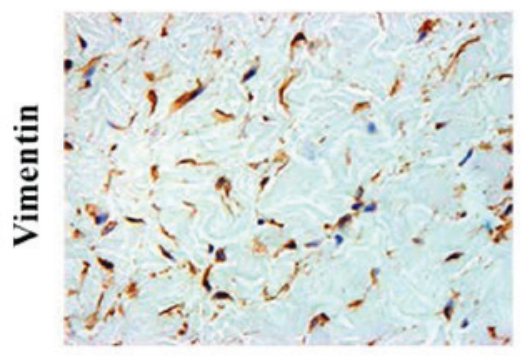

B

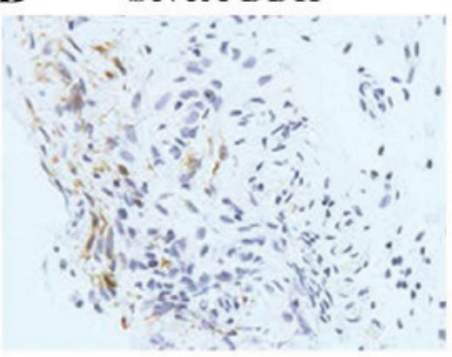

E

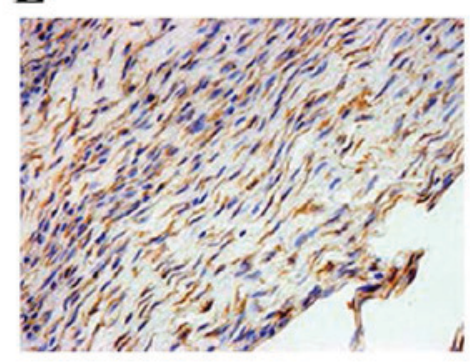

C

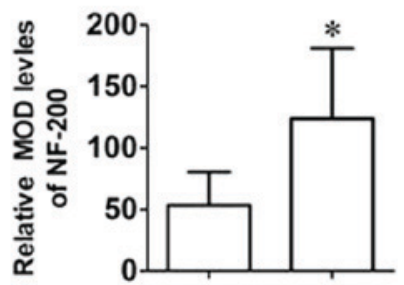

Moderate Severe $^{\text {DDH }}$

F

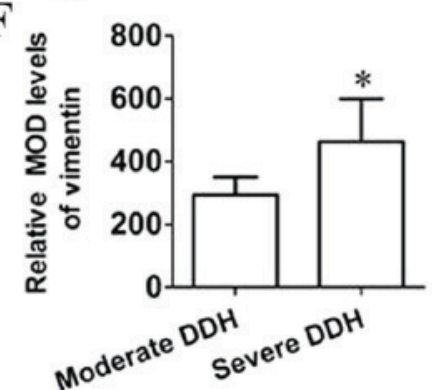

Figure 3. (A-F) Immunohistochemical staining and MOD analysis. (A, B, D and E) Immunohistochemical staining of NF-200 and Vimentin in synovium between moderate and severe DDH group. (A and B) Magnification: x400, (D and E) magnification: x200. (C and F) Comparisons of MOD levels of NF-200 and Vimentin in immunohistochemical staining between different groups. " $\mathrm{P}<0.05$ compared with moderate DDH. MOD, mean optical density; DDH, developmental dysplasia of the hip.

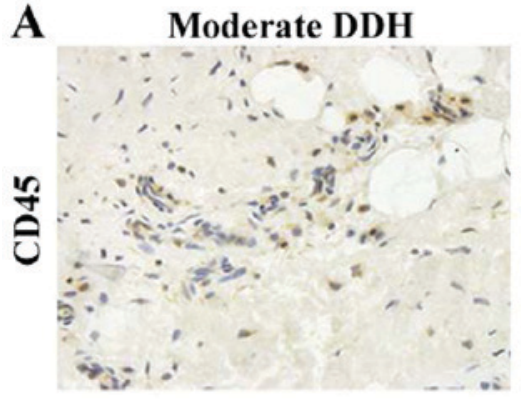

D

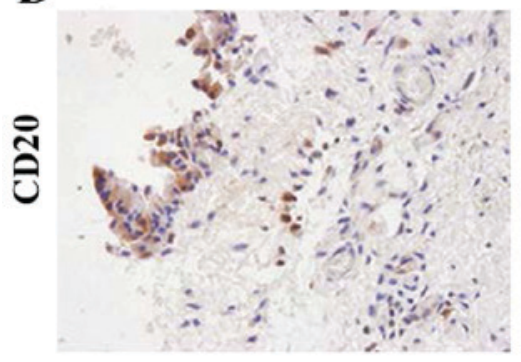

B Severe DDH

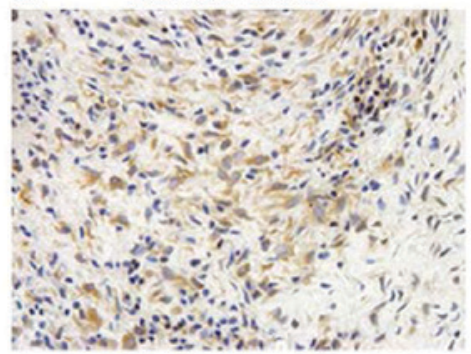

E

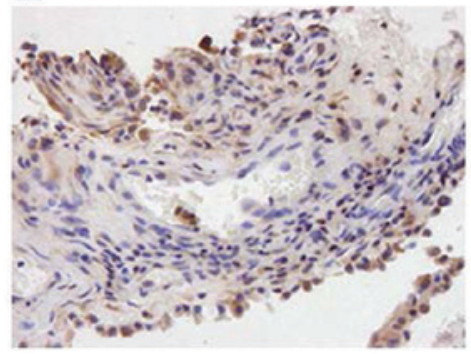

C

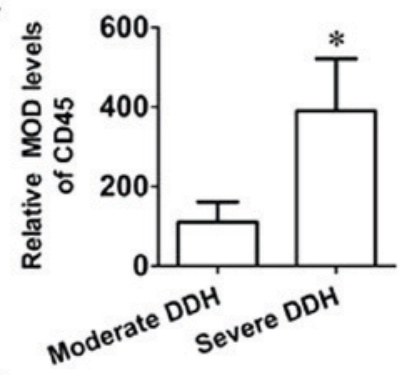

F

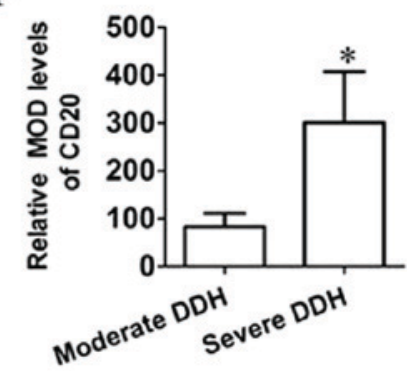

Figure 4. (A-F) Immunohistochemical staining and MOD analysis. (A, B, D and E) Immunohistochemical staining of CD45 and CD20 in synovium between moderate and severe DDH group. (A, B, D and E) Magnification: $x 400$. (C and F) Comparisons of MOD levels of CD45 and CD20 in immunohistochemical staining between different groups. ${ }^{*} \mathrm{P}<0.05$ compared with moderate DDH. MOD, mean optical density; DDH, developmental dysplasia of the hip.

\section{Discussion}

DDH is a major predisposing factor for hip OA and can be caused by improper loadings on hip joints. Owing to this reason, normal load-bearing regions in dysplasia hips, particularly articular cartilage, labrum, and synovium, are subjected to alterative mechanical overload. Among these components, the synovium in the hip joint has been widely acknowledged as the inflammation origin and an initial factor of arthritis progression in OA $(22,23)$. Therefore, considering the abnormal mechanical environment of synovium from $\mathrm{DDH}$, we deemed investigating the biological appearance of synovium from DDH in vivo necessary.

Although the application of IHC method in pain research in vivo has been widely acknowledged $(24,25)$, limited progress has been made in identifying the mechanisms of inflammatory progress of hip joint, especially the pathological changes in the synovial layer. Thus, the most significant aspect in this study was the use of IHC for hip joint synovium and the exploration of pathological changes in the synovium of $\mathrm{DDH}$, 
A Moderate DDH
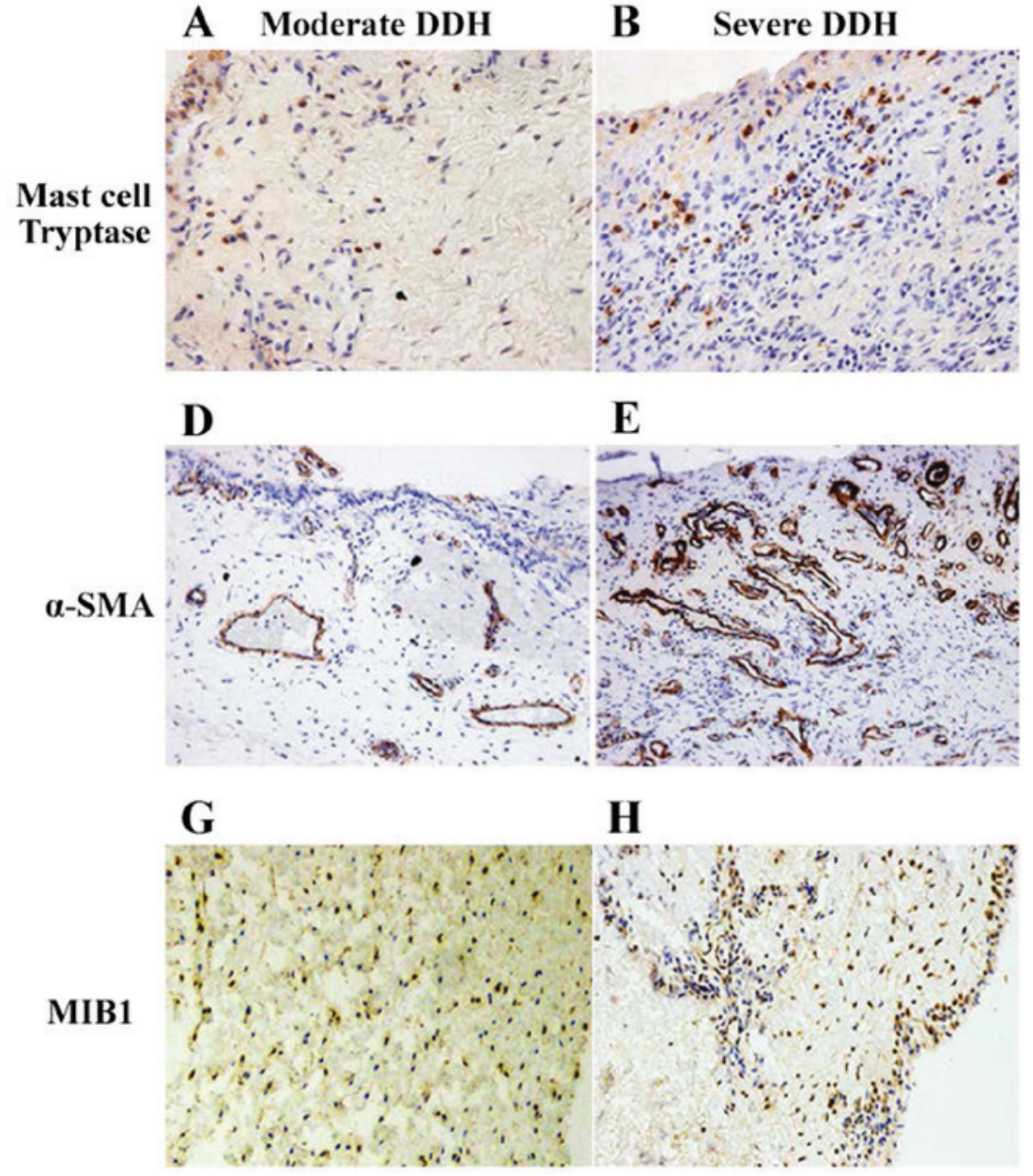

C

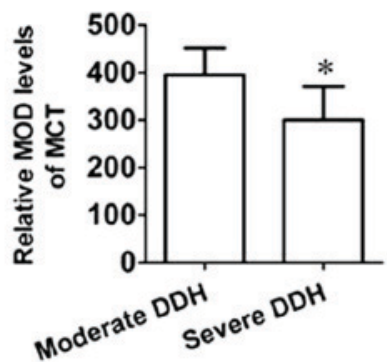

$\mathbf{F}$

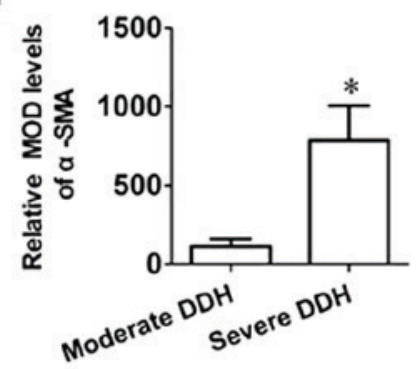

I

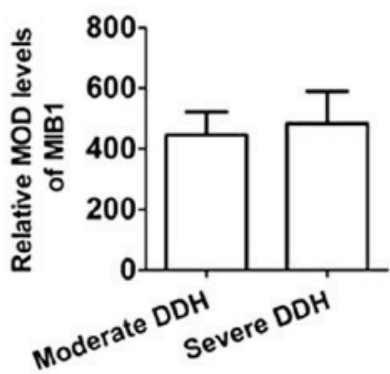

Figure 5. (A-I) Immunohistochemical staining and MOD analysis. (A, B, D, E, G and H) Immunohistochemical staining of MCT, $\alpha$-SMA and MIB1 in synovium between moderate and severe DDH group. (A, B, G and H) Magnification: x200; (D and E) magnification: x100. (C, F and I) Comparisons of MOD levels of MCT, $\alpha$-SMA and MIB1 in immunohistochemical staining between different groups. "P<0.05 compared with moderate DDH. MOD, mean optical density; DDH, developmental dysplasia of the hip; MCT, mast cell tryptase; $\alpha$-SMA, $\alpha$-smooth muscle actin; MIB1, mindbomb homolog 1.

A
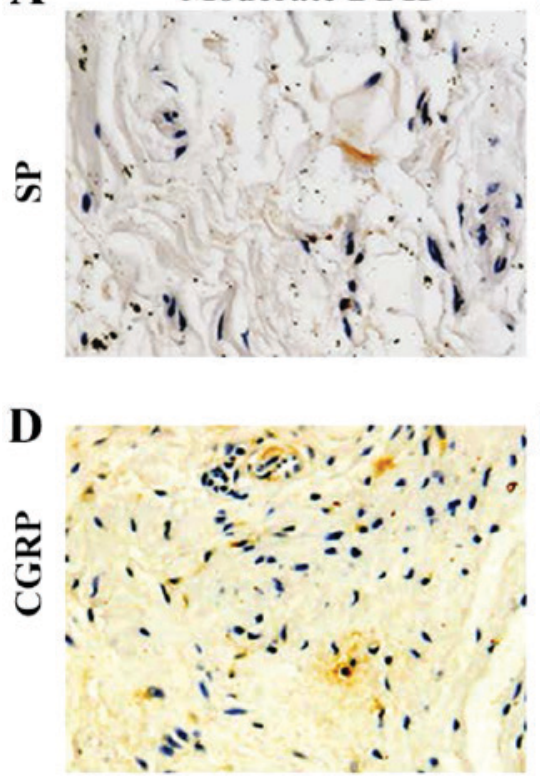

B
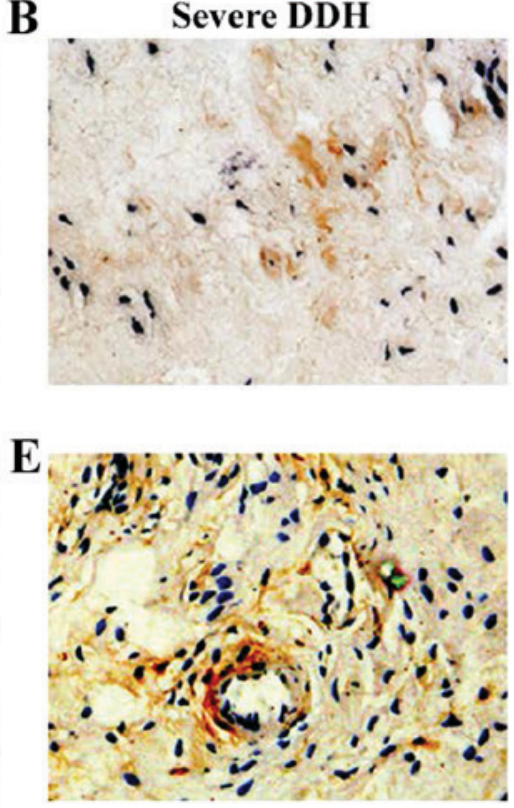

C
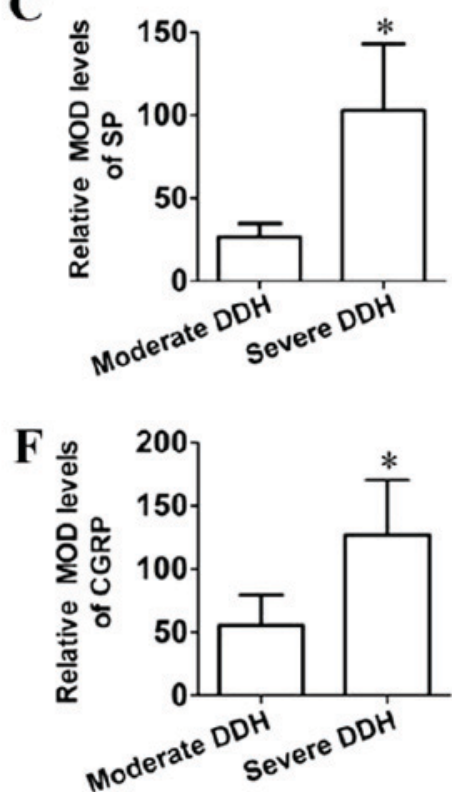

Figure 6. (A-F) Immunohistochemical staining and MOD analysis. (A, B, D and E) Immunohistochemical staining of SP, CGRP in synovium between moderate and severe DDH group. (A, B, D and E) Magnification: x400. (C and F) Comparisons of MOD levels of SP, CGRP in immunohistochemical staining between different groups. "P<0.05 compared with moderate DDH. MOD, mean optical density; DDH, developmental dysplasia of the hip; SP, substance P; CGRP, calcitonin gene-related peptide. 
Table III. Correlation analysis of MOD levels of each antibody with HHS and VAS.

\begin{tabular}{|c|c|c|c|c|c|c|c|c|}
\hline \multirow[b]{3}{*}{ Antibody } & \multicolumn{4}{|c|}{ Moderate DDH } & \multicolumn{4}{|c|}{ Severe DDH } \\
\hline & \multicolumn{2}{|c|}{ vs. HHS } & \multicolumn{2}{|c|}{ vs. VAS } & \multicolumn{2}{|c|}{ vs. HHS } & \multicolumn{2}{|c|}{ vs. VAS } \\
\hline & $\mathrm{R}$ & $\mathrm{P}$ & $\mathrm{R}$ & $\mathrm{P}$ & $\mathrm{R}$ & $\mathrm{P}$ & $\mathrm{R}$ & $\mathrm{P}$ \\
\hline \multicolumn{9}{|l|}{ Inflammatory cells } \\
\hline CD3 & 0.010 & 0.945 & 0.187 & 0.219 & -0.234 & 0.176 & 0.276 & 0.109 \\
\hline CD20 & -0.351 & 0.018 & 0.279 & 0.064 & -0.394 & 0.019 & 0.378 & 0.025 \\
\hline CD45 & -0.449 & 0.012 & 0.555 & $<0.001^{\mathrm{a}}$ & -0.101 & 0.564 & 0.233 & 0.178 \\
\hline CD68 & -0.453 & 0.018 & 0.239 & 0.113 & -0.669 & $<0.001^{\mathrm{a}}$ & 0.518 & $0.001^{\mathrm{a}}$ \\
\hline NF-200 & -0.122 & 0.425 & 0.349 & 0.029 & -0.167 & 0.339 & 0.421 & $0.009^{\mathrm{a}}$ \\
\hline$\alpha-\mathrm{SMA}$ & -0.208 & 0.169 & 0.212 & 0.163 & -0.541 & $0.005^{\mathrm{a}}$ & 0.241 & 0.164 \\
\hline MIB1 & 0.141 & 0.356 & 0.193 & 0.205 & 0.063 & 0.721 & 0.265 & 0.124 \\
\hline Vimentin & -0.368 & $0.003^{\mathrm{a}}$ & 0.234 & 0.123 & 0.479 & $0.004^{\mathrm{a}}$ & -0.015 & 0.933 \\
\hline Mast cell tryptase & -0.209 & 0.166 & 0.571 & $<0.001^{\mathrm{a}}$ & -0.344 & 0.043 & 0.062 & 0.725 \\
\hline \multicolumn{9}{|l|}{ Neuropeptides } \\
\hline SP & -0.091 & 0.551 & 0.208 & 0.171 & -0.349 & 0.039 & 0.433 & $0.009^{\mathrm{a}}$ \\
\hline CGRP & -0.059 & 0.697 & 0.237 & 0.117 & -0.333 & 0.051 & 0.455 & $0.006^{\mathrm{a}}$ \\
\hline
\end{tabular}

MOD, mean optical density; IHC, immunohistoche-mical; SP, substance P; CGRP, calcitonin gene-related peptide; MIB1, mindbomb homolog 1; $\alpha$-SMA, $\alpha$ smooth muscle actin; R, correlation coefficient. Spearman correlation test was implemented to investigate the possible correlations in separate groups. ${ }^{a} \mathrm{P}<0.01$ means significant correlation.

which are useful in detecting the mechanism of progression from moderate to severe DDH. In this study, patients with DDH were divided into two groups (moderate and severe $\mathrm{DDH})$ to investigate the differences of pathophysiological process and inflammatory conditions between these groups in the development from moderate to severe DDH. In clinical practice, we observed that the durations of severe DDH is shorter than that of moderate DDH. As we know, the patients with severe DDH cannot tolerate the inconvenience caused by joint dysfunction. Therefore, the patients will resort to surgery as early as possible to improve hip function and relieve pain. It seemed to us that there might be advantages in an earlier surgical treatment.

The results of the histological analysis revealed an upgrade of inflammatory infiltration into the synovium, which was confirmed by IHC using a variety of antibodies. Analysis with CD3, CD20, CD45, CD68, and mast cell tryptase antibodies identified these cells to be predominantly $\mathrm{T}$ cells, B cells, leukocytes, macrophages, and mast cells, indicating it to be a chronic inflammatory cell infiltration. Among these, the MOD levels of CD45, mast cell tryptase, and CD68 showed significant correlations with VAS in moderate and severe DDH separately, which illustrate the infiltration of leukocytes, macrophage, and mast cells might be associated with the pain sensation of hip joint in DDH. Similarly, the MOD levels of vimentin, $\alpha$-SMA, and CD68 also presented significant negative correlations with HHS separately, which means the infiltration of fibroblast, myofibroblast, and macrophage probably contribute to the disorders of hip joint in the development of DDH.

The results of IHC also demonstrate the remarkable up-regulation of SP, CGRP, and NF-200 in synovium from severe DDH cases. But, no such remarkable up-regulation was observed in moderate DDH cases. This process shows the increasing infiltration of free nerve endings into the synovium during the process from moderate DDH to severe DDH. Moreover, the MOD levels of NF-200, SP and CGRP revealed positive correlation with VAS in patients with severe DDH, and such correlation emphasizes the crucial roles of free nerve endings and neuropeptides in the sensation of pain. Previous studies proved that with the stimuli of peripheral pain, SP and CGRP were increasingly synthesized in dorsal root ganglion (DRG) then secreted by peripheral sensory nerve endings (26). Basing on our findings, we speculate that with the time and duration of DDH, cartilage wearing degree will gradually increase, and the cushion capacity of cartilage progressively disappears. Therefore, the subchondral bone and labrum tissue bear increasing shear and compressive loadings as the cartilage abrasion evolves. During this process, the afferent stimulation of joint pain gradually increases, and this increase in turn induce the infiltration of peripheral nerve fibers (NF-200) and is accompanied by the release of additional neuropeptides (SP and CGRP) from the DRG.

As emphasized in our previous studies, SP and CGRP might have the pro-inflammatory effect on synoviocyte from DDH through the activation of NF- $\kappa \mathrm{B}$ (16). Moreover, according to previous studies, the activation of NF- $\kappa \mathrm{B}$ in synoviocyte could induce the release of chemokines, matrix metalloproteinases-1 (MMP-1), MMP-3 and interleukins-6 (IL-6) (27,28). Among these, chemokines such as monocyte chemotactic protein-1 (MCP-1) and macrophage inflammatory protein-1 (MIP-1 $\beta$ ) are entrusted with the task of recruiting macrophage as well as other monocytes for involvement in the inflammatory 
process of synovium. Hence, we speculate that the abrasion of cartilage might stimulate the release of SP and CGRP from the nerve fibers to the synovial tissues during the progress of $\mathrm{DDH}$, and this condition can aggravate the degeneration of cartilage in DDH by recruiting macrophages and other inflammatory effector cells from peripheral blood. Moreover, these inflammatory effector cells can secrete a series of harmful cytokines (MMPs and ILs etc.), which can further accelerate the destruction of the cartilage of DDH.

Synovial fibrosis in DDH was explored by the positive staining of $\alpha$-SMA (myofibroblast) and vimentin (fibroblast) in the synovium. Previous research reported the extracellular matrix (ECM) exocrine function and biological activity of myofibroblast. As the indicator of vascular smooth muscle and angiogenesis, the appearance of myofibroblast was acknowledged indispensable in fibrosis and chronic inflammation (29,30). Fibroblast dedicates autonomous contributions in the inflammatory process as effector cells releasing pro-inflammatory mediators and undertaking antigen presenting effect by expressing MHC-II and auto-antigens to specific T cell hybridomas (31-34). Thus, in this study, the increasing IHC staining of $\alpha$-SMA and vimentin in synovium combined with the positive correlations of these targets with HHS and VAS imply the existence of fibrosis in synovium and its harm to joint motion.

Moreover, as a hallmark of OA, synovial fibrosis is characterized by fibroblast proliferation and imbalance between collagen synthesis and catabolism (35). This imbalance results in the excessive deposition of collagen into ECM leading to thickening and stiffening of the synovial membrane, which is believed to be a major contributor to joint pain and stiffness. This basic theory has been verified by the positive correlations of these fibrosis elements ( $\alpha$-SMA and vimentin) with clinic indexes (HHS). Therefore, these synovial fibrosis related indexes could be promising therapy targets, which probably help to reduce fibrosis and stiffness of hip joint in DDH.

The current understanding of the involvement of mast cells in inflammation included leukocyte recruitment and activation, angiogenesis, fibroblast proliferation, matrix remodeling, and injury to collagen and bone $(36,37)$. Herein, the presence of mast cells in synovium in this study makes us speculate the involvement of mast cells in both chronic inflammation and synovium fibrosis, which probably lead to secondary injury and destruction of cartilage. By its pivotal position in the inflammatory process, the mast cells may also hold promise as a treatment target to relieve the inflammatory progress of arthritis in DDH.

The biopsy material from the synovia of patients with DDH with different stages revealed that this disorder is a chronic inflammation and fibrotic condition. Together with the presence of extensive angiogenesis and nerve fibers, this ascending inflammation and fibrosis indicated from IHC in synovium probably explains why the upgrade of DDH could combine with an increasingly painful and stiff syndrome. Targeted prevention of synovial inflammatory infiltration and fibrosis could relief the degeneration of cartilage and restrain the progress of DDH.

\section{Acknowledgements}

Not applicable.

\section{Funding}

This study was supported by the National Natural Science Foundation of Youth in China (grant nos. 81101381 and 81601866), Three-year planning for promoting clinical skills and innovation in municipal hospitals by develop-center of Shanghai Shenkang Hospital (grant no. 16CR2036B) and Foundation of Shanghai Municipal Health Bureau (grant no. 20134002).

\section{Availability of data and materials}

The analyzed data sets generated during the study are available from the corresponding author on reasonable request.

\section{Authors' contributions.}

DL participated in the design of the study, image processing, and manuscript preparation and revision. HW performed immunohistochemistry and revised the manuscript. JYH performed the questionnaires of VAS and HHS. CLW and WJF participated in immunohistochemistry and data statistical analysis. CS performed statistical analysis and manuscript revision. JFZ participated in the collection of synovial biopsies from patients. XDC and DLW conceived the study, participated in its design and coordination, and gave general supervision of the whole research group. All authors have read and approved the final manuscript.

\section{Ethics approval and consent to participate}

The implementation of this study was based on the recommendations from the Declaration of Helsinki and was approved by the ethical committee of Shanghai Jiaotong University School Medicine Affiliate Xinhua Hospital (Approval no. XHEC-D-2013-009). The samples obtained from the operations were prepared for testing purposes only and with the approval and signed consent of the patients.

\section{Patient consent for publication}

Not applicable.

\section{Competing interests}

The authors declare that they have no competing interests.

\section{References}

1. Lisle R, Boekelaar M, Stannage K and Whitewood C: Delayed diagnosis of developmental dislocation of the hip: The Western Australian experience. ANZ J Surg 82: 612-615, 2012.

2. Shi D, Dai J, Zhu P, Qin J, Zhu L, Zhu H, Zhao B, Qiu X, Xu Z, Chen D, et al: Association of the D repeat polymorphism in the ASPN gene with developmental dysplasia of the hip: A case-control study in Han Chinese. Arthritis Res Ther 13: R27, 2011.

3. Sangal RB, Waryasz GR and Schiller JR: Femoroacetabular impingement: A review of current concepts. R I Med J (2013) 97: 33-38, 2014.

4. Alzaharani A, Bali K, Gudena R, Railton P, Ponjevic D, Matyas JR and Powell JN: The innervation of the human acetabular labrum and hip joint: An anatomic study. BMC Musculoskelet Disord 15: 41, 2014. 
5. Henak CR, Abraham CL, Anderson AE, Maas SA, Ellis BJ, Peters CL and Weiss JA: Patient-specific analysis of cartilage and labrum mechanics in human hips with acetabular dysplasia. Osteoarthritis Cartilage 22: 210-217, 2014.

6. Haversath M, Hanke J, Landgraeber S, Herten M, Zilkens C, Krauspe $\mathrm{R}$ and Jäger $\mathrm{M}$ : The distribution of nociceptive innervation in the painful hip: A histological investigation. Bone Joint J 95-B: 770-776, 2013.

7. Nakajima T, Ohtori S, Inoue G, Koshi T, Yamamoto S, Nakamura J, Takahashi K and Harada Y: The characteristics of dorsal-root ganglia and sensory innervation of the hip in rats. J Bone Joint Surg Br 90: 254-257, 2008.

8. Henrotin Y, Lambert C and Richette P: Importance of synovitis in osteoarthritis: Evidence for the use of glycosaminoglycans against synovial inflammation. Semin Arthritis Rheum 43: 579-587, 2014

9. Henrotin Y, Pesesse L and Lambert C: Targeting the synovial angiogenesis as a novel treatment approach to osteoarthritis. Ther Adv Musculoskelet Dis 6: 20-34, 2014.

10. Shirai C, Ohtori S, Kishida S, Harada Y and Moriya H: The pattern of distribution of PGP 9.5 and TNF-alpha immunoreactive sensory nerve fibers in the labrum and synovium of the human hip joint. Neurosci Lett 450: 18-22, 2009.

11. Takeshita M, Nakamura J, Ohtori S, Inoue G, Orita S, Miyagi M, Ishikawa T and Takahashi K: Sensory innervation and inflammatory cytokines in hypertrophic synovia associated with pain transmission in osteoarthritis of the hip: A case-control study. Rheumatology (Oxford) 51: 1790-1795, 2012.

12. Beckmann J, Schubert J, Morhenn HG, Grau V, Schnettler R and Lips KS: Expression of choline and acetylcholine transporters in synovial tissue and cartilage of patients with rheumatoid arthritis and osteoarthritis. Cell Tissue Res 359: 465-477, 2015.

13. de Lange-Brokaar BJ, Ioan-Facsinay A, van Osch GJ, Zuurmond AM, Schoones J, Toes RE, Huizinga TW and Kloppenburg M: Synovial inflammation, immune cells and their cytokines in osteoarthritis: A review. Osteoarthritis Cartilage 20: 1484-1499, 2012.

14. Kim YS, Kim JM, Lee YG, Hong OK, Kwon HS and Ji JH: Intercellular adhesion molecule-1 (ICAM-1, CD54) is increased in adhesive capsulitis. J Bone Joint Surg Am 95: e181-e188, 2013

15. Hand GC, Athanasou NA, Matthews T and Carr AJ: The pathology of frozen shoulder. J Bone Joint Surg Br 89: 928-932, 2007.

16. Wang H, Zhang X, He JY, Zheng XF, Li D, Li Z, Zhu JF, Shen C, Cai GQ and Chen XD: Increasing expression of substance P and calcitonin gene-related peptide in synovial tissue and fluid contribute to the progress of arthritis in developmental dysplasia of the hip. Arthritis Res Ther 17: 4, 2015.

17. Crowe JF, Mani VJ and Ranawat CS: Total hip replacement in congenital dislocation and dysplasia of the hip. J Bone Joint Surg Am 61: 15-23, 1979.

18. Hart DJ and Spector TD: The classification and assessment of osteoarthritis. Baillieres Clin Rheumatol 9: 407-432, 1995.

19. Cashman JP, Round J, Taylor G and Clarke NM: The natura history of developmental dysplasia of the hip after early supervised treatment in the Pavlik harness. A prospective, longitudinal follow-up. J Bone Joint Surg Br 84: 418-425, 2002.

20. Nakamura S, Ninomiya $S$ and Nakamura T: Primary osteoarthritis of the hip joint in Japan. Clin Orthop Relat Res: 190-196, 1989.
21. Rondelet B, Kerbaul F, Motte S, van Beneden R, Remmelink M, Brimioulle S, McEntee K, Wauthy P, Salmon I, Ketelslegers JM and Naeije R: Bosentan for the prevention of overcirculation-induced experimental pulmonary arterial hypertension. Circulation 107: 1329-1335, 2003.

22. O'Neill TW, Parkes MJ, Maricar N, Marjanovic EJ, Hodgson R, Gait AD, Cootes TF, Hutchinson CE and Felson DT: Synovial tissue volume: A treatment target in knee osteoarthritis (OA). Ann Rheum Dis 75: 84-90, 2016.

23. Liu-Bryan R: Synovium and the innate inflammatory network in osteoarthritis progression. Curr Rheumatol Rep 15: 323 , 2013.

24. Adães S, Mendonça M, Santos TN, Castro-Lopes JM, Ferreira-Gomes $\mathbf{J}$ and Neto FL: Intra-articular injection of collagenase in the knee of rats as an alternative model to study nociception associated with osteoarthritis. Arthritis Res Ther 16: R10, 2014.

25. Daghestani HN, Pieper CF and Kraus VB: Soluble macrophage biomarkers indicate inflammatory phenotypes in patients with knee osteoarthritis. Arthritis Rheumatol 67: 956-965, 2015.

26. Zhang RX, Ren K and Dubner R: Osteoarthritis pain mechanisms: Basic studies in animal models. Osteoarthritis Cartilage 21: 1308-1315, 2013.

27. Wakamatsu K, Nanki T, Miyasaka N, Umezawa K and Kubota T: Effect of a small molecule inhibitor of nuclear factor-kappaB nuclear translocation in a murine model of arthritis and cultured human synovial cells. Arthritis Res Ther 7: R1348-R1359, 2005.

28. Laragione T and Gulko PS: Liver X receptor regulates rheumatoid arthritis fibroblast-like synoviocyte invasiveness, matrix metalloproteinase 2 activation, interleukin- 6 and CXCL10. Mol Med 18: 1009-1017, 2012.

29. Mifková A, Kodet O, Szabo P, Kučera J, Dvořánková B, André S, Koripelly G, Gabius HJ, Lehn JM and Smetana K Jr: Synthetic polyamine BPA-C8 inhibits TGF- $\beta 1$-mediated conversion of human dermal fibroblast to myofibroblasts and establishment of galectin-1-rich extracellular matrix in vitro. Chembiochem 15: 1465-1470, 2014.

30. Minton K: Extracellular matrix: Preconditioning the ECM for fibrosis. Nat Rev Mol Cell Biol 15: 766-767, 2014.

31. Shinde AV and Frangogiannis NG: Fibroblasts in myocardial infarction: A role in inflammation and repair. J Mol Cell Cardiol 70: 74-82, 2014

32. Leech MT and Morand EF: Fibroblasts and synovial immunity. Curr Opin Pharmacol 13: 565-569, 2013.

33. Sobel K, Tham M, Stark HJ, Stammer H, Prätzel-Wunder S, Bickenbach JR and Boukamp P: Wnt-3a-activated human fibroblasts promote human keratinocyte proliferation and matrix destruction. Int J Cancer 136: 2786-2798, 2015.

34. Kägebein D, Gutjahr M, Große C, Vogel AB, Rödel J and Knittler MR: Chlamydia trachomatis-infected epithelial cells and fibroblasts retain the ability to express surface-presented major histocompatibility complex class I molecules. Infect Immun 82: 993-1006, 2014.

35. Scanzello CR and Goldring SR: The role of synovitis in osteoarthritis pathogenesis. Bone 51: 249-257, 2012.

36. Nigrovic PA and Lee DM: Synovial mast cells: Role in acute and chronic arthritis. Immunol Rev 217: 19-37, 2007.

37. da Silva EZ, Jamur MC and Oliver C: Mast cell function: A new vision of an old cell. J Histochem Cytochem 62: 698-738, 2014. 